\title{
An online financial accounting system for Chinese colleges and universities in the Internet environment
}

\author{
Hu Ouzhe $e^{1, a,{ }^{*}}$ \\ ${ }^{1}$ Department of Finance, Wuhan University of Technology, Wuhan 430070, Hubei, China \\ ahuouzhe@163.com \\ ${ }^{*}$ Corresponding author
}

Keywords: financial accounting system, online, colleges and universities.

\begin{abstract}
The current backward financial management system of Chinese colleges and universities is in urgent need of transformation. An online financial accounting system for Chinese colleges and universities in the Internet environment is proposed in this paper. The system model includes user layer, front-end business layer, core business layer, business support layer and data server layer. Each layer is composed of different business modules. On this basis, a new and efficient business process has been established. Application example shows that the system is effective and helpful.
\end{abstract}

\section{Introduction}

At present, as the research funding of Chinese colleges and universities increases year by year, the work pressure of the traditional finance department is getting bigger and bigger [1]. Their work efficiency becomes low, and various contradictions are more glaring than ever [2].

There are many reasons for this result. Due to the staffing constraints, the number of personnel in the finance department is unlikely to increase, and the demand for business volume cannot be fully met. Moreover, the functions of existing financial software are also very limited, and the scalability is poor, resulting in a time difference in the update of financial data $[3,4,5]$. Not only can teachers not know the use progress of the project's funding in a timely manner, but also there is a difference between the details of the funds' use obtained by the various departments of the school on their own authorized information terminals and financial information that is settled in real time by the finance department [6, 7]. As a result, some projects will not meet the corresponding schedule requirements for use of funds within the specified time limit, and the remainder was eventually recovered by the state, which will result in the reduction of project allocation in the coming year. In addition, the lag and non-uniformity of financial information is also not conducive to the scientific decision-making of school leaders.

Changing the reimbursement service model and improving efficiency are the fundamental way to alleviate existing contradictions. With the rapid development of computer technology and the wide application of network technology in people's daily life, human beings are gradually entering the era of network big data from the industrial age [8,9]. This paper will present an online financial management method for Chinese colleges and universities under the background of the Internet, which can solve the existing problems well and realize an ideal model for all data to be unified, shared, and real-time.

\section{System model and business process}

\subsection{System model}

In view of the existing financial management problems in Chinese colleges and universities at present, a new solution is proposed in this paper based on the practical situation. The system model is shown in Fig. 1. 


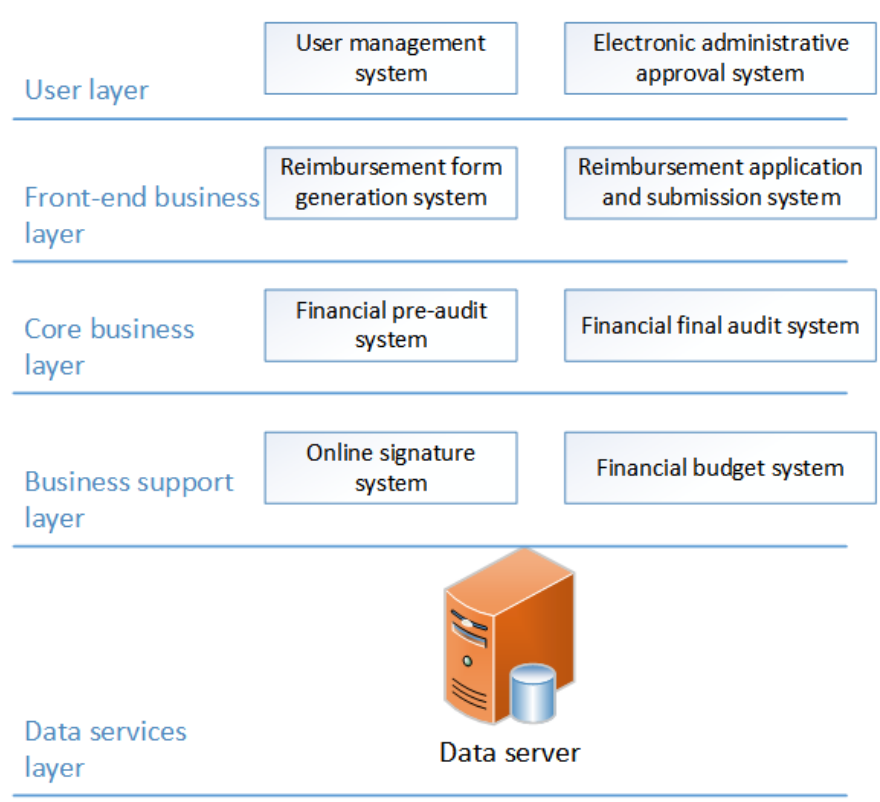

Fig.1 System model

The system is divided into five layers, namely user layer, front-end business layer, core business layer, business support layer and data server, and each layer is composed of different business modules.

The user layer includes a user management system and an electronic administrative approval system. The user management system is responsible for user management and provides a login interface. The reimbursement applicant logs in to the system with the user name (personnel number or job number) and password to implement the authorization operation. The electronic administrative approval system is mainly responsible for providing online credible project leaders and supervisors to approve and realize electronic signature and electronic official seal.

The front-end business layer includes the reimbursement form generation system and the reimbursement application and submission system. The reimbursement form generation system is responsible for the generation of the reimbursement form. The reimbursement applicant fills in the fund item, the reimbursement amount and the bank account according to the reimbursement information, and a reimbursement form for the corresponding type will be automatically generated after the completion of the filling. The reimbursement application and submission system is mainly responsible for the online receiving and management of reimbursement applications.

The core business layer includes the financial pre-audit system and the financial final audit system. The financial pre-audit system conducts preliminary pre-audit of the compliance and accuracy of the information in the reimbursement application, and prepares for the reimbursement process to enter the final step. The financial final audit system is responsible for the final reimbursement work, and will cooperate with the business support layer to conduct the final audit. If it is qualified, the reimbursement business will be completed, otherwise it will be canceled.

The business support layer includes the online signature system and the financial budget system. The online signature system realizes the management and validity examination of electronic signature, which can prevent the occurrence of business fraud. The financial budget system ensures that the overall reimbursement is automatically carried out according to the project's preliminary budget, which can effectively prevent the occurrence of over-budget behavior.

The data server layer mainly contains data servers and provides data storage and retrieval services for the whole system through Internet network. It is a key infrastructure layer in the background.

\subsection{Business process}

The system composed of the above modules can efficiently realize the financial accounting and management of Chinese colleges and universities. The overall business process is shown in Fig. 2. 


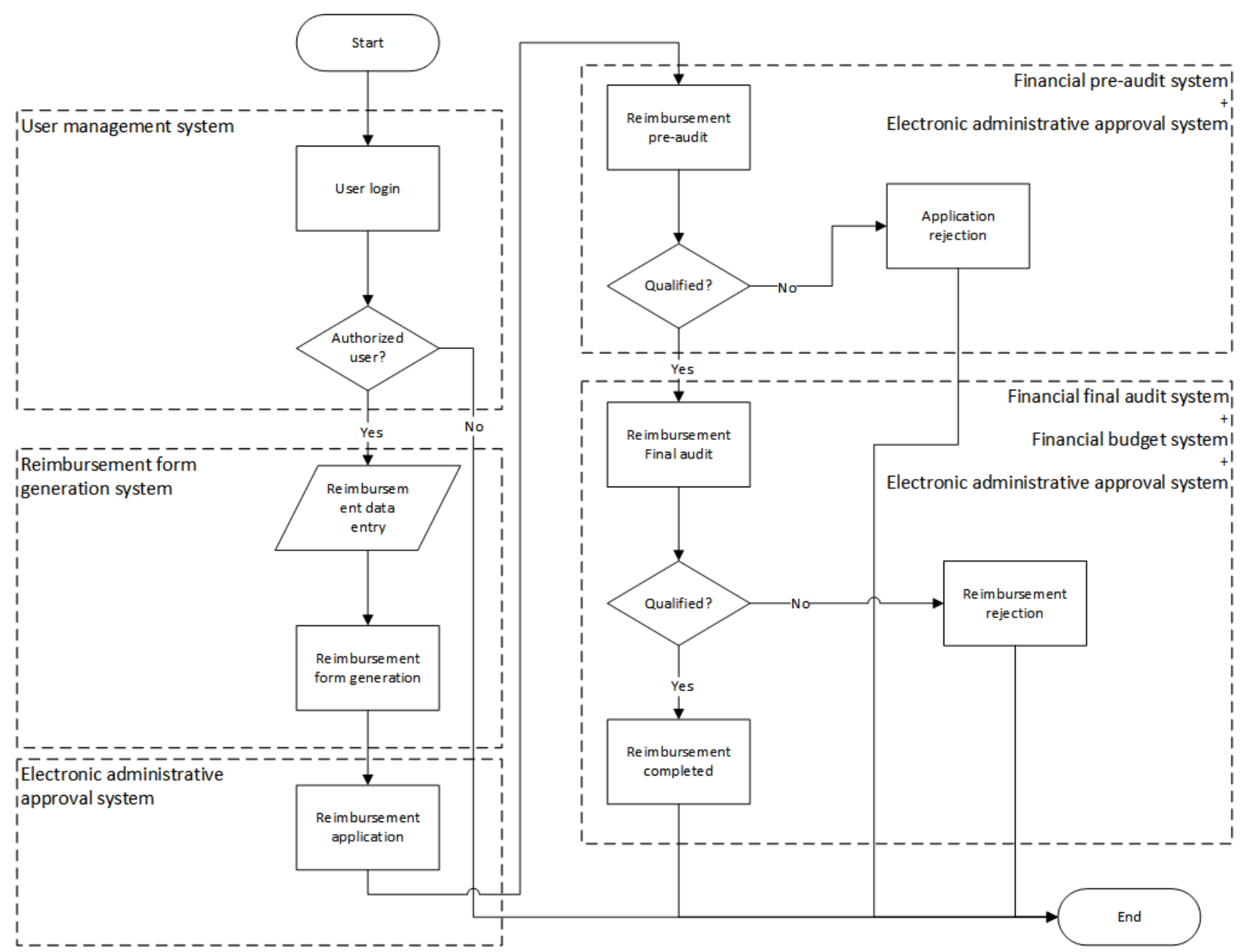

Fig. 2 Business process and relations with basic modules

As can be seen from Figure 2, the reimbursement applicant first implement login authentication through the user login system, and qualified users can enter the next process. Then it enters the stage of data collection. The reimbursement applicant fills in the data information such as the expense item, reimbursement content, reimbursement amount and bank account according to the reimbursement tips, and then generates the reimbursement form. After the reimbursement form is generated, the reimbursement applicant submits the application for reimbursement, and the reimbursement form enters the electronic administrative approval system, the administrative examination and approval procedure will start according to the sequence. After the completion of the electronic administrative examination and approval, the reimbursement applicant will submit the reimbursement form and the original vouchers to the financial pre-audit personnel. After the financial pre-audit is passed, the final audit procedure will be initiated. The financial final audit system will be combined with the financial budget system and electronic signature system to carry out the final verification. If qualified, the system will complete the reimbursement process and update all data on the back-end data server.

The overall logic of the above business process is clear. All parts of the system can work well together, and the data consistency is also very good, so the overall efficiency is very high.

\section{Example of application}

The above system was tested in a university. Based on its existing software and hardware, we updated and reconfigured its backstage data server, and improved its financial pre-audit and final audit system, and reimbursement form generation system, newly added online signature system and electronic administrative approval system, and then realized the connection with its financial budget system. At the same time, we also redesigned its business process.

The application of this system has greatly improved the financial operation process of the university, and its benefits are mainly as follows: 
(1) Reimbursement efficiency has increased more than five times.

(2) Provide more timely financial information, and build an effective communication bridge between the reimbursement applicants and financial staff.

(3) Connect to the budget system in real time, effectively prevented the use of funds in excess of scope and budget.

(4) Provide online signature and approval function, which greatly saves the time required for the entire process.

(5) The data is timely and consistent, and financial reports are easier to obtain, which provides a powerful help for financial decision-making.

\section{Summary}

At present, the traditional financial management mode of Chinese colleges and universities is facing great challenges. It is necessary to transform it with computer technology and network technology. In this paper, an effective attempt for online financial management models and methods in Chinese universities is provided. The application example shows that this new management system and its business process can greatly improve the financial management of Chinese colleges and universities, and it is very efficient and helpful.

\section{References}

[1] Zhang Qiaohua, Solve the Problem of College Reimbursement from the Perspective of Process Reengineering. Business Accounting, vol.1, pp. 19-23, 2015.

[2] Yin Tao, Research on the Reform of Financial Reimbursement Work in Colleges and Universities under the "Internet +" Mode, Education Finance Research Report, vol.12, pp. 17-21, 2015.

[3] Zhang Qiaohua, Research on University Network Reimbursement Model of Financial Informatization, Modern Business, vol.32, pp. 15-18, 2014.

[4] Luo Yanqin, thinking about the difficulty of reimbursement for college funds, Finance and Accounting Monthly, vol.17, pp. 37-41, 2016.

[5] Xie Wei, A Brief Account of College Reimbursement Management and Process - Take B Medical School as an Example, Business Accounting, vol.21, pp. 24-27, 2015.

[6] Sheng Qingyan, Research on the Financial Reimbursement Information System Model of Colleges and Universities under the Background of Internet +, China Science and Technology Information, vol.15, pp. 50-53, 2016.

[7] Qiu Juncheng, Theoretical Research and Practical Exploration of Online Reimbursement in Colleges and Universities, Friends of Accounting, vol.10, pp. 19-23, 2017.

[8] Luo Jun, Xie Yulong, Research on the Management of Financial Accounting in Colleges and Universities under the "Internet +" Environment, Cooperative Economy and Technology, vol.15, pp. 17-21, 2017.

[9] Chen Yongjun, Applied Research on the "Internet +" University Financial Reimbursement Model, Modern Economic Information, vol.22, pp. 33-37, 2017. 